ORIGINAL ARTICLE

\title{
Effect of socioeconomic status on objectively measured physical activity
}

\section{A Kelly, J J Reilly, A Fisher, C Montgomery, A Williamson, J H McColl, J Y Paton, S Grant}

See end of article for authors' affiliations

Correspondence to:

Dr J J Reilly, Reader in

Paediatric Energy

Metabolism, University

Division of Developmental

Medicine, 1st Floor, Tower

Block QMH, Yorkhill

Hospitals, Dalnair Street,

Glasgow G3 8SJ, UK;

iir2y@clinmed.gla.ac.uk

Accepted 11 October 2005

Published Online First

20 October 1005

Arch Dis Child 2006;91:35-38. doi: 10.1136/adc.2005.080275

Background: A socioeconomic gradient in childhood obesity is known to be present by the age of school entry in the UK. The origin of this gradient is unclear at present, but must lie in socioeconomic differences in habitual physical activity, sedentary behaviour, or dietary intake.

Aims: To test the hypothesis that habitual physical activity and/or sedentary behaviour are associated with socioeconomic status (SES) in young Scottish children.

Methods: Observational study of 339 children (mean age 4.2 years, SD 0.3) in which habitual physical activity and sedentary behaviour were measured by accelerometry over six days (study 1). In a second study, 39 pairs of children of distinctly different SES (mean age 5.6 years, SD 0.3) were tested for differences in habitual physical activity and sedentary behaviour by accelerometry over seven days.

Results: In study 1, SES was not a significant factor in explaining the amount of time spent in physical activity or sedentary behaviour once gender and month of measurement were taken into account. In study 2 , there were no significant differences in time spent in physical activity or sedentary behaviour between affluent and deprived groups.

Conclusion: Results do not support the hypothesis that low SES in young Scottish children is associated with lower habitual physical activity or higher engagement in sedentary behaviour.
$\mathrm{T}$ he obesity epidemic has affected even preschool children, ${ }^{12}$ and in the developed world, has had a disproportionately greater effect on more deprived families. ${ }^{3}$ This socioeconomic gradient is evident by the preschool years and persists into later childhood and adolescence. $^{245}$

Obesity is the result of a chronic energy imbalance (an excess of intake over expenditure), but the origin of socioeconomic differences in this imbalance is unclear. A systematic review concluded that the evidence for socioeconomic status (SES) differences in risk factors for obesity and heart disease in childhood was inconclusive, and more research was required. ${ }^{6}$ This review included many older studies, studies which were probably underpowered, studies which did not measure physical activity objectively, and studies which did not consider sedentary behaviour. Marked socioeconomic differences in energy intake are not evident in dietary surveys in British children, ${ }^{2}$ but dietary intake assessment tends to be inaccurate and imprecise, even in children. ${ }^{7}$ With the advent of accelerometry, physical activity and sedentary behaviour (a distinct construct from physical activity which might be particularly important to obesity $\operatorname{risk}^{8}$ ) can be measured objectively in free-living children with relatively high precision and accuracy. Hypothesis testing as to the origin of early SES differences in obesity risk might, therefore, usefully be focused on measurement of physical activity and sedentary behaviour with accelerometry. Accordingly, the aim of the present study was to test whether there were significant differences in objectively measured habitual physical activity and sedentary behaviour in young children in relation to SES.

\section{SUBJECTS AND METHODS}

\section{Overall design}

We carried out two studies to test the hypothesis that habitual physical activity and/or sedentary behaviour differed between SES groups. In the first, we carried out an observational study (study 1) of SES and habitual physical activity and sedentary behaviour in children participating in the baseline stage of a randomised controlled trial, the "MAGIC" study. ${ }^{89}$ In the second study, children from families of high and low SES were paired (to control for gender, age, season, and school days/school holidays), and habitual physical activity and sedentary behaviour were compared between the two groups (study 2).

\section{Subjects}

Study 1

We measured physical activity and sedentary behaviour in preschool children (mean age 4.2 years, SD 0.5), with approximately equal numbers of children measured during the months of September and October 2002. ${ }^{\circ}$ Children $(\mathrm{n}=382)$ were randomly selected for physical activity measurements from the larger sample participating in the baseline measurements of a randomised controlled trial $(\mathrm{n}=545),{ }^{9}$ and were eligible for the present study if they provided at least six hours per day of accelerometry over six days ( $\mathrm{n}=339$ of the 382 eligible). The children studied were broadly representative of Glasgow in terms of SES. ${ }^{9}$ We assessed SES using the Carstairs score, a geographically based measure (based on a composite of social class, car ownership, unemployment, and overcrowded housing). ${ }^{10}$ This is a standard index of SES in Scotland, and has been shown to reflect marked differences in socioeconomic circumstances and health outcomes. ${ }^{10}{ }^{11}$ The Carstairs score uses seven categories, from 1 (most affluent) to 7 (most deprived). For study 1 , we collapsed the seven categories to three (groups $1-2 ; 3-5 ; 6-7)$ and had physical activity data for 20,93 , and 226 children in each of the three groups respectively.

\section{Study 2}

We recruited two groups of children of distinct SES, an affluent and deprived group (Carstairs scores $1-2$ and 6-7 respectively). Children were recruited from year 1 of two 
distinct types of primary school: local authority (for Carstairs groups 6 and 7) and private sector schools (for Carstairs groups 1 and 2). Recruitment of children in this way not only facilitated selection of two distinct socioeconomic groups, but the differences in education (local authority versus private) reinforced the impression that these two groups were actually socioeconomically distinct. Recruitment produced 116 children potentially available for inclusion. We included all children in deprivation categories 1 and 2 (affluent group) and deprivation categories 6 and 7 (deprived group) whom we could match pair-wise for gender and school days/school holidays; our sample consisted of 78 children (39 pairs; 20 pairs of girls, 19 pairs of boys; mean age 5.6 years, SD 0.4 ) for study 2, all of whom provided at least six hours of accelerometry per day over seven days during November.

For both studies 1 and 2, all children were apparently healthy, with no chronic disease relevant to energy balance or physical activity. The studies were approved by the Yorkhill Hospitals Ethics Committee. Informed written consent was obtained from the parent/guardian of each child.

\section{Measurements of habitual physical activity and sedentary behaviour}

In study 1 we measured physical activity and sedentary behaviour objectively over six days during the waking hours (mean duration of measurement 54.9 hours, SD 13.8) using accelerometers. In study 2 we measured the same variables over seven days using the same methods (mean duration of measurement 73.9 hours, SD 12.3). In both studies we asked families to attach the accelerometers when the children woke up, to remove them when they went to bed, and to record when and why they were removed at other times. The accelerometers were set to monitor activity in one minute sampling intervals (epochs) as previously described. ${ }^{12}$ These activity monitoring periods exceed the time required to determine usual physical activity and sedentary behaviour. ${ }^{13}$ We have previously observed negligible day-to-day variation (for example, weekday-weekend variation) in accelerometry output in our samples of young children in Scotland, and no systematic within-child, within-day variation in accelerometry output (time of day effects). ${ }^{12}$

Accelerometry count averaged over the monitoring period (count per minute, cpm) was used as an index of total physical activity; ${ }^{12}{ }^{14}$ we also calculated percentage of monitored time spent sedentary (no trunk movement, $<1100 \mathrm{cpm}){ }^{15}$ and percentage of monitored time in moderate-vigorous physical activity (MVPA; $>3200 \mathrm{cpm}^{16}$ ). These definitions or "cut points" for accelerometry output have been validated against both energy expenditure ${ }^{16}$ and direct observation of behaviour ${ }^{15}$ in previous studies.

\section{Statistical analysis \\ Study 1}

Analysis of variance and covariance models were used to assess the effect of the following explanatory variables on mean accelerometry output (cpm): age; BMI SD score; gender; SES; month of measurement. For each of these variables separately a univariate analysis was carried out. A final multivariate model was obtained by backward stepwise elimination from a model including all the five explanatory variables.

\section{Study 2}

Since study 2 used a paired design we tested differences between the two SES groups for significance using paired statistical methods. Our power calculation estimated that (with a paired design) a mean difference in total physical activity of around $100 \mathrm{cpm}$ between groups would be detectable with $90 \%$ power at a significance 0.05 , in $38-40$
Table 1 Characteristics of participants, physical activity, and sedentary behaviour

\begin{tabular}{|c|c|c|}
\hline Characteristic & $\begin{array}{l}\text { Study } 1 \\
(n=339)\end{array}$ & $\begin{array}{l}\text { Study } 2 \\
(n=78)\end{array}$ \\
\hline Age (years) & $4.2(0.3)$ & $5.6(0.4)$ \\
\hline BMI SD score & $0.40(0.89)$ & $0.61(0.73)$ \\
\hline$\%$ time spent sedentary* & 77 (53-93) & $78(67-90)$ \\
\hline $\begin{array}{l}\% \text { time spent in moderate-vigorous } \\
\text { physical activity* }\end{array}$ & $3(0-13)$ & $3(1-9)$ \\
\hline
\end{tabular}

pairs of children. This magnitude of difference is approximately $10-15 \%$ of accelerometry output since this usually averages $700-800 \mathrm{cpm}^{12}{ }^{14}{ }^{17}$ For context, this difference in physical activity is of a similar magnitude to that typically observed between the sexes at this age. ${ }^{12} 1417$

\section{RESULTS}

Physical characteristics of subjects, levels of physical activity, and sedentary behaviour

The physical characteristics of children in both studies 1 and 2 are shown in table 1 .

\section{Study 1: Factors influencing physical activity}

The results of the analysis are shown in table 2. A univariate analysis found that mean accelerometry output (cpm) was significantly lower in the most affluent SES group compared to the other two groups, but this association did not persist once other variables were taken into account in the multivariate analysis. Only gender and month of measurement were included in the final model in study 1 . Total physical activity was significantly higher in boys than girls, and significantly higher in September than October.

\section{Study 2: Differences in total physical activity between socioeconomic groups}

Total physical activity (accelerometry, cpm) did not differ significantly on average for affluent and deprived participants. Mean accelerometry count per minute in the affluent participants was $734 \mathrm{cpm}$ (SD 151) compared with $793 \mathrm{cpm}$ (SD 196) for the deprived participants (paired $t$ test, $\mathrm{p}=0.10)$. There were also no statistically significant differences in total physical activity between affluent and deprived participants when considered separately by gender $(p>0.05$ in each case).

\section{Study 2: Differences in moderate-vigorous intensity physical activity between socioeconomic groups}

Median percentage of monitored time spent in moderate to vigorous intensity activity (MVPA) was identical in the affluent and deprived participants: 3\% (range $1-7$ ) versus 3\% (range 1-9) respectively.

\section{Study 2: Differences in sedentary behaviour between socioeconomic groups}

Median percentage of monitored time spent in sedentary behaviour was not significantly different for the two groups (affluent participants, 79\% (range 68-90) versus deprived participants, 78\% (range 67-89); Wilcoxon sign rank test, $\mathrm{p}=0.13$ ).

When data were analysed separately by gender, we found that in boys, percentage time spent in sedentary behaviour was significantly lower in the deprived than the affluent group (Wilcoxon sign rank test, $\mathrm{p}=0.01$ ). For all other analyses of physical activity and sedentary behaviour, results did not differ when analysed with the entire group or by gender. 
Table 2 Analysis of variance and covariance for study 1 (total physical activity, mean accelerometer count per minute)

\begin{tabular}{|c|c|c|c|c|}
\hline \multirow[b]{2}{*}{ Explanatory variable } & \multicolumn{2}{|c|}{ Univariate analysis } & \multicolumn{2}{|c|}{ Multivariate analysis } \\
\hline & Coefficient (ESE) & $p$ value & Coefficient (ESE) & $p$ value \\
\hline Gender, female $v$ male & $-62.0(20.7)$ & 0.003 & $-59.1(20.3)$ & 0.004 \\
\hline Month (Oct v Sept) & $-83.9(20.7)$ & $<0.0005$ & $-81.8(20.5)$ & $<0.0005$ \\
\hline SES, $1 \vee 3$ & $-124.5(44.6)$ & 0.02 & - & - \\
\hline SES, $2 \vee 3$ & $-1.8(23.6)$ & NS & - & - \\
\hline Age (years) & $16.3(30.1)$ & NS & - & - \\
\hline BMI SD score & $17.4(11.2)$ & NS & - & - \\
\hline
\end{tabular}

$\mathrm{R}^{2}$ for final model, $7.0 \%$.

ESE, estimated standard error; NS, not significant.

\section{DISCUSSION}

In the present study we found no evidence of marked differences in either habitual physical activity or sedentary behaviour between SES groups. Thus, our observations do not support the hypotheses that more deprived children are less physically active or more sedentary than more affluent children, despite marked differences in obesity risk associated with SES. ${ }^{245}$ Our failure to observe differences in physical activity and sedentary behaviour in the predicted direction could relate to lack of power, but with the paired design, study 2 was powered to detect quite small differences in engagement in total physical activity $(100 \mathrm{cpm}$ between groups)—differences that may be too small to be biologically meaningful. ${ }^{12} 141517$ Study I was one of the largest studies to use objective methods to measure physical activity in preschool children to date.

The present study suggests that social patterning of childhood obesity in the UK may be due to socioeconomic differences in dietary intake rather than differences in habitual physical activity or sedentary behaviour. This perspective on the aetiology of obesity is reductionist as it considers the origin of obesity purely from the point of view of energy balance, the difference between energy input and output. We accept that the aetiology of obesity is more complex than this in reality, and that research which considers the many possible behavioural or biological determinants of socioeconomic differences in obesity will be helpful in providing an improved understanding of its social patterning in future.

At present very few studies have reported on objectively measured physical activity and sedentary behaviour in young children. The determinants of physical activity in childhood remain unclear, ${ }^{18}{ }^{19}$ and SES has not emerged strongly as a predictor of physical activity in the literature. ${ }^{19}$ Predictors of objectively measured sedentary behaviour in young children are even less well understood since this topic has been little researched, only emerging in the literature as an important variable relatively recently. ${ }^{8} 15$

The cut-offs we applied to accelerometry output to determine time spent in different intensities of activity have been validated against direct observation of behaviour and energy expended on activity in children in independent studies, ${ }^{15} 16$ and so should have provided accurate measurement of time spent sedentary and in MVPA. However, it is possible that subtle differences in the types of activity undertaken by children in different socioeconomic groups, not measured by accelerometry, might be important. It is also possible that habits associated with sedentary behaviour (such as eating/snacking while watching TV) ${ }^{20}$ might have differed between the socioeconomic groups, but these would not have been identifiable using accelerometry. Short sleep duration has recently been suggested as a possible risk factor for childhood obesity, ${ }^{21}$ and it is conceivable that this might differ between socioeconomic groups, but we did not set out to measure sleep duration precisely in the present study, or to power our study to detect differences in sleep duration between groups.

In the present study we considered habitual physical activity and sedentary behaviour as separate constructs, as is now the norm. ${ }^{8}{ }^{15}$ However, we accept that in circumstances where one form of behaviour (in this case sedentary behaviour) predominates, this may displace opportunities for other forms of behaviour such as moderate-vigorous physical activity. ${ }^{14} 1722$

It is possible that there are differences in habitual physical activity between ethnic groups in the UK, or that relations between physical activity and adiposity might differ between ethnic groups, or between boys and girls. Children who participated in the present studies were almost entirely from the majority ethnic group in Scotland, and so we could not address the issue of ethnicity. We found evidence that social patterning of sedentary behaviour might differ between boys and girls in study 2, but this needs to be confirmed in future studies in other samples and settings. One recent study found a possible gender difference in the relation between physical activity and adiposity in 7 year old British children. ${ }^{22}$

In the present studies, we used a uniaxial accelerometer (designed to measure activity predominantly in the vertical plane). While in theory measurement in two or three planes of movement might provide greater accuracy, empirical studies comparing uniaxial versus biaxial or triaxial accelerometry do not support this. ${ }^{23}{ }^{24}$ We summarised accelerometry output in one minute measurement intervals (epochs). In theory, shorter epochs might provide more accurate quantification of more vigorous activities, but again empirical tests do not support this hypothesis, and use of shorter measurement intervals does not provide an advantage in practice. ${ }^{25}$ The main practical impact of this approach to the interpretation of accelerometry output appears to be a small systematic misclassification of some vigorous activity as moderate intensity activity; ${ }^{25}$ this is one reason why we summarised both categories in combination.

\section{Conclusions}

The results of the present studies are not consistent with the hypothesis that differences in physical activity or sedentary behaviour underlie socioeconomic variations in obesity risk in early childhood. The present study leads to the prediction that socioeconomic differences in dietary intake underlie the social patterning of obesity in young British children.

\section{ACKNOWLEDGEMENTS}

Study 1 was funded by the British Heart Foundation. We thank Glasgow City Council for their support, the schools who helped with recruitment to study 2 , and the parents and children who took part for their enthusiastic participation. 


\section{What is already known on this topic}

- Children from more socioeconomically deprived families are at much greater risk of obesity than those from wealthier families

- The reasons for this social patterning in childhood obesity are unclear, but must lie in socioeconomic differences in physical activity, dietary intake, or both

\section{What this study adds}

- Habitual physical activity is not influenced by socioeconomic status

- Socioeconomic differences in obesity risk are likely to be due to social patterning of dietary intake

\section{Authors' affiliations}

L A Kelly, J J Reilly, A Fisher, C Montgomery, J Y Paton, University of Glasgow Division of Developmental Medicine, Yorkhill Hospitals, Glasgow, UK

S Grant, University of Glasgow Faculty of Biological and Life Sciences, West Medical Building, Glasgow, UK

A Williamson, Glasgow City Council Education Department, Wheatley House, Merchant City, Glasgow, UK

J H McColl, University of Glasgow Department of Statistics, Glasgow, UK Competing interests: none

\section{REFERENCES}

1 Reilly JJ, Dorosty AR. Epidemic of obesity in UK children. Lancet 1999;354:1874-5.

2 Armstrong J, Dorosty AR, Reilly JJ, Child Health Information Team, Emmett PM. Co-existence of social inequalities in undernutrition and obesity in preschool children: population based cross sectional study. Arch Dis Child 2003:88:671-5.

3 Lobstein T, Baur L, Uauy R. Obesity in children and young people: a crisis in public health. Obesity Reviews 2004;5(suppl):4-85.
4 Armstrong J, Reilly JJ, Child Health Information Team. Prevalence of obesity and underweight in Scottish children: growth monitoring within the Child Health Surveillance Programme. Scot Med J 2003;48:32-7.

5 Kinra S, Nelder RP, Lewendon GJ. Deprivation and childhood obesity: a crosssectional study of 20,973 children in Plymouth, UK. J Epidemiol Community Health 2000;54:456-60.

6 Batty GD, Leon DA. Socio-economic position and coronary heart disease risk factors in children and young people. Eur J Public Health 2002;12:263-72.

7 Reilly JJ, Montgomery C, Jackson D, et al. Energy intake by multiple pass 24hour recall and total energy expenditure. Br J Nutr 2001;86:601-5.

8 Reilly JJ, McDowell ZC. Physical activity interventions in the prevention and treatment of childhood obesity: systematic review and critical appraisal. Proc Nutr Soc 2003:62:61 1-19.

9 Fisher A, Reilly JJ, Kelly LA, et al. Fundamental movement skills and habitual physical activity in young children. Med Sci Sports Exerc 2005;37:684-8.

10 Carstairs V, Morris R. Deprivation and health in Scotland. Aberdeen: Aberdeen University Press, 1991.

11 Chalmers J Capewell S. Deprivation, disease, and death in Scotland: graphical display of survival of a cohort. BMJ 2001;323:967-8.

12 Jackson DM, Reilly JJ, Kelly LA, et al. Objectively measured physical activity and inactivity in 3-4 year olds. Obes Res 2003;11:420-5.

13 Trost SG, Pate RR, Freedson PS, et al. Using objective physical activity measures with youth: how many days of monitoring are needed? Med Sci Sports Exerc 2000;31:426-31.

14 Reilly JJ, Jackson D, Montgomery C, et al. Total energy expenditure and physical activity in young Scottish children: mixed longitudinal study. Lancet 2004;363:211-12

15 Reilly JJ, Coyle J, Kelly LA, et al. An objective method for measurement of sedentary behavior in 3-4 year olds. Obes Res 2003;11:1155-8.

16 Puyau MR, Adolph AL, Firoz AV, et al. Validation and calibration of activity monitors in children. Obes Res 2002;10:150-7.

17 Montgomery C, Reilly JJ, Jackson DM, et al. Relation between physical activity and energy expenditure in a representative sample of young children. Am J Clin Nutr 2004;80:591-6.

18 Finn KJ, Johannsen N, Specker B. Factors associated with physical activity in pre-school children. J Pediatr 2002;140:81-5.

19 Sallis JF, Prochaska JJ, Taylor WC. A review of correlates of physical activity of children and adolescents. Med Sci Sports Exerc 2000;32:963-75.

20 Robinson TN. Reducing children's TV viewing to prevent obesity: a randomised controlled trial. JAMA 1999;282:1561-7.

21 Reilly JJ, Armstrong J, Dorosty AR, ALSPAC Study Team, et al. Early life risk factors for obesity in contemporary children: cohort study. BMJ 2005;330: 1357-62

22 Metcalf BS, Voss LD, Mallam KM, et al. What children do all day: the physical activity of 7 year olds and its impact on adiposity, Obes Rev 2005;114:381.

23 Welk GJ, Corbin CB. The validity of the tritrac R-3D activity monitor for assessment of physical activity. Res Q Exerc Sport 1995;66:202-9.

24 Kelly LA, Reilly JJ, Fairweather SC, et al. Comparison of two accelerometers for assessment of physical activity in pre-school children. Pediatr Exerc Sci 2004; 16:324-33.

25 Nilsson A, Ekelund U, Yngve A, et al. Assessing physical activity among children with accelerometers using different time sampling intervals and placement. Pediatr Exerc Sci 2002;14:87-96. 